\section{Coating Materials Containing Gold Nanoparticles}

\author{
Ayako Iwakoshi, Taizo Nanke and \\ Toshikatsu Kobayashi \\ Corporate Research Laboratory, \\ Research and Development, Nippon Paint Co., Ltd. \\ 19-17, Ikedanaka-machi, Neyagawa, Osaka, 572-8501, \\ Japan \\ E-mail: iwakoshi_np6958@npc.nipponpaint.co.jp \\ URL: http://www.nipponpaint.co.jp/
}

\begin{abstract}
Highly concentrated pastes of gold nanoparticles were successfully produced by using a special type of protective polymer and a mild reductant. The solid part of these pastes comprises up to $85 \mathrm{wt} \%$ of gold nanoparticles, $10 \sim 30 \mathrm{~nm}$ in diameter and a residual protective polymer. This component can be diluted at arbitrary rates with any type of solvent by selecting the polarity of the protective polymer. Electronics, optics, catalysis, colourants and surface metal coatings are the possible fields of application of these pastes.
\end{abstract}

Key words: gold, nanoparticle, colourant, coating, paint
Noble metal nanoparticles are of interest due to their various applications, such as colourants, metal coating, electronics (1), optics (2) and chemical catalysis (3).

Particularly, in the field of colours, gold nanoparticles are commonly used as an aesthetic red colourant for stained glasses and fine glassware, such as a Venetian glass. The mechanism of colouring is attributed to the surface plasmon absorption of gold nanoparticles (4).

The gold nanoparticles used in the colouring of a stained glass exhibit a distinct red colour, which does not fade for hundreds of years. On the other hand, the red colour of the organic pigments in a paint film often fades in several years. We intend to reproduce the elegant and stable colouring of a stained glass in paint films. In order to colour a very thin, 20 50 $\mu \mathrm{m}$, paint film in the manner of stained glass, the production of highly concentrated dispersed gold nanoparticles is required. In order to achieve this, two issues need to be resolved. The first issue is the manner in which the coagulation of gold nanoparticles in a highly concentrated solution can be prevented. A lot of methods to produce gold nanoparticles (5) have been reported thus far. However, only a few of these methods have successfully stabilized the gold nanoparticle dispersion at concentrations higher than the order of ppm. Second issue deals with the control of the particle size. This is because the colour of gold nanoparticles is very sensitive to the particle size. The suspension exhibits red colour like a stained glass only when the particle size is around 10nm.

We have developed a novel method to produce a uniformly dispersed, concentrated and stable dispersion of gold nanoparticles having diameters in the range of 10 30 $\mathrm{nm}$ (6). This method comprises of two key technologies. The first is the prevention of particles from mutual coagulation. In order to accomplish this, we used a special type of combshaped block copolymer to stabilize the particles (Figure 1). The polymers have multiple amino groups on the main chain

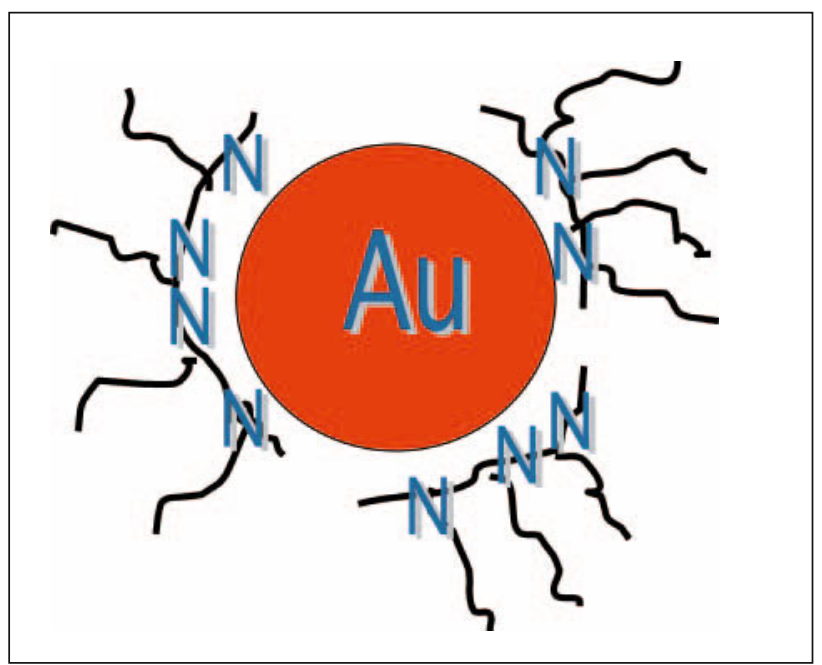

Figure 1

Comb-shaped Block Copolymer Stabilized Gold Nanoparticle 
and side chains with different chemical structures. The second is concerned with the control of particle size, that is, the reduction of gold ions. We observed that tertiary amines mildly reduce gold ions and yield nanoparticles under industrially controllable conditions.

A combination of these two key technologies enabled us to produce stable and highly concentrated gold nanoparticle pastes. Thus far, we have succeeded in producing pastes containing up to $85 \mathrm{wt} \%$ gold particles in the solid part. These pastes can be diluted in a stable manner or mixed with various types of aqueous and non-aqueous media by selecting the polarity of the side chain of the protective polymer.

In this paper, we will introduce two applications of our gold nanoparticle pastes. One of them is that of colourants. When a small amount of gold nanoparticle paste is added to a polymer or glassy matrix, it gives a transparent, clear red colour. Another application is that of thin metal film production. Since the metal content in the solid part was very high, we expected that a simple coating and baking procedure could yield a thin metal film.

\section{Experimental}

Tetrachloroauric (III) acid tetrahydrate $\left(\mathrm{HAuCl}_{4} \cdot 4 \mathrm{H}_{2} \mathrm{O}\right)$ was purchased from Tanaka Kikinzoku Kogyo K.K. and was used without further purification.

A water-soluble polyacrylate-based comb-shaped block copolymer (Polymer-W) was used to produce aqueous gold nanoparticle pastes. The amounts of acid and base evaluated by potentiometric titration were 0.53 and $0.37 \mathrm{mmol} / \mathrm{g}$, respectively. A polyester-based comb-shaped block copolymer (Polymer-S) was used for the solvent type pastes. The amounts of acid and base were 0.58 and $0.64 \mathrm{mmol} / \mathrm{g}$, respectively. The molecular weight of both the polymers was several tens of thousand. As schematically shown in Figure 1, Polymer-W and Polymer-S possess secondary or tertiary amino groups in the

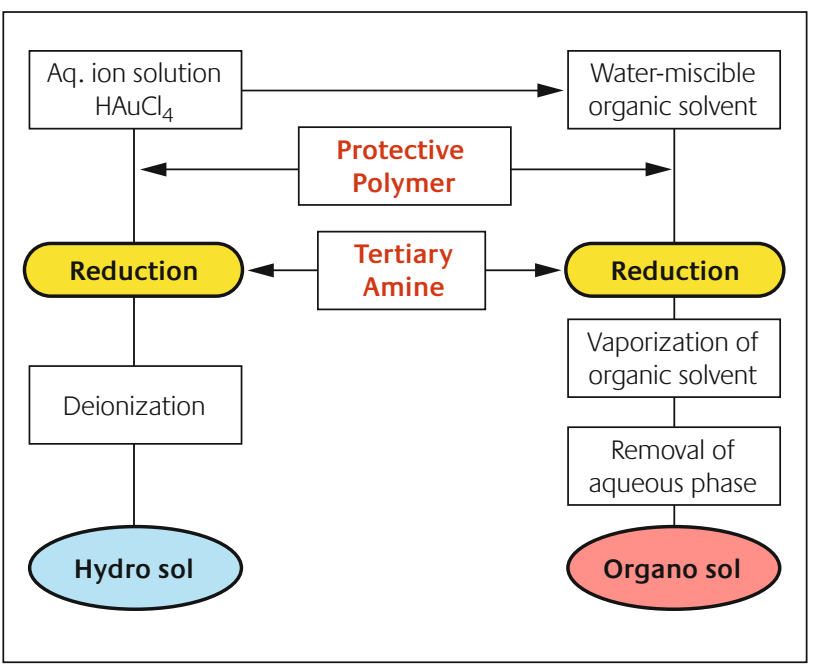

Figure 2

Typical Procedure for Preparation of Gold Nanoparticle Pastes main chain and were expected to adsorb on the gold nanoparticles by these amino groups.

Reagent grade of N,N'-dimethylethanolamine (Kishida Chemical Co.) was used without further purification for the reduction of $\mathrm{Au}^{3+}$ ion.

Reagent grades of ethanol, acetone and toluene (all from Kishida Chemical Co.) and ion-exchanged water were used as solvents for the reaction and dilution of the pastes and paints.

\subsection{Production of concentrated gold nanoparticle pastes}

Gold nanoparticle pastes were produced by the reduction of $\mathrm{HAuCl}_{4}$ with tertiary amine in the presence a protective polymer. A typical procedure of the production is as follows (Figure 2).

\section{1) Hydro sol (Aqueous type pastes; AuE-001 and AuW-001)}

$27 \mathrm{~g}$ of $\mathrm{HAuCl}_{4} \cdot 4 \mathrm{H}_{2} \mathrm{O}$ was dissolved in $230 \mathrm{~g}$ of $\mathrm{H}_{2} \mathrm{O}$. Further, $19 \mathrm{~g}$ of Polymer-W was added and stirred till a uniform solution was obtained. The mixture was heated up to $50^{\circ} \mathrm{C}$ and then $29 \mathrm{~g}$ of 2-dimethylaminoethaol was added. The solution was then stirred for $2 \mathrm{~h}$. This crude colloidal dispersion was deionised by ultrafilteration (Microza'M UF AHP-1010, Asahi Kasei Co.) with ethanol (for AuE-001) or ionexchanged water (for AuW-001) to produce the red gold nanoparticle pastes.

\section{2) Hydro sol (Aqueous type pastes; AuE-101 and AuW-101)}

$40 \mathrm{~g}$ of $\mathrm{HAuCl}_{4} \cdot 4 \mathrm{H}_{2} \mathrm{O}$ was dissolved in $747 \mathrm{~g}$ of $\mathrm{H}_{2} \mathrm{O}$. Further, $8.2 \mathrm{~g}$ of Polymer-W was added and stirred till the solution became uniform. The mixture was heated up to $50^{\circ} \mathrm{C}$. Then, $43 \mathrm{~g}$ of 2 -dimethylaminoethaol was added. The solution was stirred for $2 \mathrm{~h}$. This crude colloidal dispersion was deionised by ultrafilteration (MicrozaTM UF AHP-1010, Asahi Kasei Co.) with ethanol (for AuE-101) or ion-exchanged water (for AuW101) to produce the gold nanoparticle pastes.

\section{3) Organo sol (Solvent type paste; AuT-001)}

$12 \mathrm{~g}$ of $\mathrm{HAuCl}_{4} \cdot 4 \mathrm{H}_{2} \mathrm{O}$ was dissolved in $40 \mathrm{~g}$ of $\mathrm{H}_{2} \mathrm{O}$. Further, $4 \mathrm{~g}$ of Polymer-S was dissolved in $60 \mathrm{~g}$ of acetone. The polymer solution was added to the $\mathrm{HAuCl}_{4}$ solution and stirred till the solution became uniform. $13 \mathrm{~g}$ of 2-Dimethylaminoethaol was added at room temperature. After the mixture was stirred for $1 \mathrm{~h}$, acetone was removed from the crude colloidal dispersion by vaporization. The solid component containing gold nanoparticles began to settle as the acetone was removed. After the solid component completely settled, the aqueous phase was removed by decantation and the residual sediment was again dissolved into toluene.

\section{4) Measurement}

1. Metal concentration

The proportion of solid content in the pastes was determined 
by the ordinary gravitational method. This involves the removal of solvents by heating the solution at $140^{\circ} \mathrm{C}$ for $1 \mathrm{~h}$. The metal concentration in the solid part of the pastes was evaluated using a thermo-gravimetric/differential thermal analyzer (TG-DTA 220, Seiko Instruments). The solid part of the pastes (ca. $10 \mathrm{mg}$ ) was heated from room temperature to $500^{\circ} \mathrm{C}$ at a rate of $10^{\circ} \mathrm{C} / \mathrm{min}$. Earlier experiments have revealed that both Polymer-W and Polymer-S completely degrade between $250-450^{\circ} \mathrm{C}$.

\section{Optical properties}

Light absorption spectra for 10000-time-diluted-pastes were recorded by a UV-Visible/NIR spectrophotometer (U-3500 Spectrophotometer, Hitachi). Ion-exchanged water and toluene were used to dilute the aqueous and solvent type pastes, respectively.

\section{Particle size}

The particle size was measured by a transmission electron microscopy (TEM) (JEM-2000 FXII Electron Microscope, JEOL).

\subsection{Application as colourants}

\section{i. Gold nanoparticle paint}

For a nanoparticle paint, a polyester polymer (Polymer-S) was used as a protective polymer. The paste was mixed with an acrylic-melamine resin system to prepare the thermosetting nanoparticle paint. The particle concentration in the solid components was $0.5 \sim 4 \%$ by weight. The nanoparticle paint was used as a 'color clear coating'. First, a base coating containing aluminum flakes was applied on a primed steel substrate. Then, the nanoparticle paint was applied on it (Figure 3). After baking the coatings at $140^{\circ} \mathrm{C}$ for $20 \mathrm{~min}$, the appearance of the coatings was visually inspected. Flip-flop effect (7) of the coating was measured by a goniophotometer (GCMS-4, Murakami Color Research Laboratory). The resistance of the paint to weather was assessed by the exposure test in Okinawa.

\section{ii. Coloured glass film}

Gold nanoparticle paste was mixed with a sol-gel binder system to prepare a thin glass film containing gold nanoparticles. The particle concentration in the solid components was $2 \%$ by weight. The sol-gel binder containing gold nanoparticles was spin-coated on the glass.

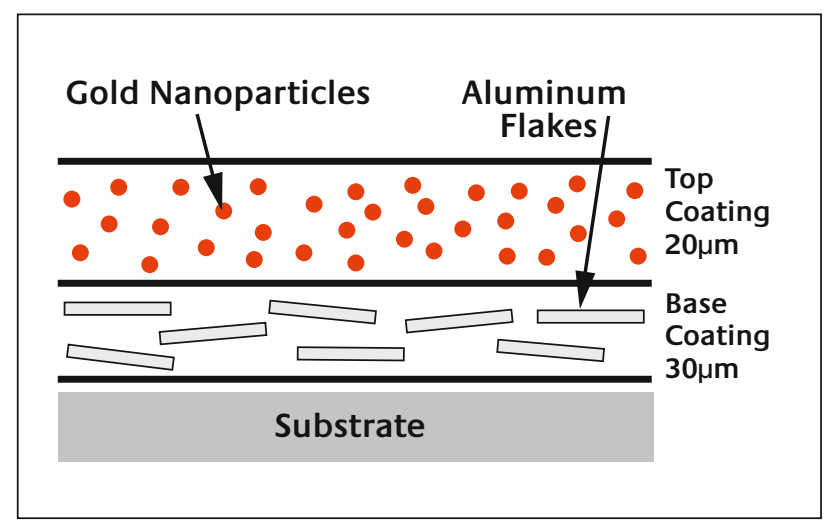

Figure 3

Cross Section of Gold Nanoparticle Paint

The appearance of the coatings was visually inspected after drying at $140^{\circ} \mathrm{C}$ for $10 \mathrm{~min}$ and baking at $250^{\circ} \mathrm{C}$ for $1 \mathrm{~h}$. The heat resistance of this glass film was measured at $280^{\circ} \mathrm{C}$.

\subsection{Application to thin metal film production}

For the thin metal film preparation, an acrylic copolymer (Polymer-W) was used as a protective polymer. Since the polarity of the polymer was higher than that of the polyester polymer used in the colourants application, the pastes were soluble in polar solvents, such as water and $C_{1} \sim C_{3}$ alcohols. For this usage, the gold content in the solid should be over $80 w t \%$.

The paste was diluted by ethanol to achieve on appropriate viscosity so as to coat it on substrates. Airspraying, spin-coating, dip-coating and inkjet printing methods were used for coating. Glass plates, PET films, china figures and glossy photo paper were used as substrates. The coated films were dried at room temperature and their appearance was visually inspected.

\section{$3 \quad$ Results and Discussion}

\subsection{Gold nanoparticle pastes}

The gold nanoparticle paste was successfully produced with almost $100 \%$ yield by all the methods described in 2.1. The compositions of the gold nanoparticle pastes, which are currently available, are shown in Table 1. The protective polymer can possess various types of chemical structures in

Table 1

Composition of Gold Nanoparticle pastes

\begin{tabular}{|c|c|c|c|c|c|}
\hline & Solvent & $\begin{array}{l}\text { Gold particle } \\
\text { size }\end{array}$ & $\begin{array}{l}\text { Gold content } \\
\text { in solid }\end{array}$ & $\begin{array}{l}\text { Polymer content } \\
\text { in solid }\end{array}$ & $\begin{array}{l}\text { Solid (gold + polymer) } \\
\text { concentration in paste }\end{array}$ \\
\hline AuE-001 & Ethanol & \multirow{3}{*}{$10-15 \mathrm{~nm}$} & \multirow{3}{*}{$70 w t \%$} & \multirow{3}{*}{$30 w t \%$} & \multirow{5}{*}{ Up to $50 w t \%$} \\
\hline AuW-001 & Water & & & & \\
\hline AuT-001 & Toluene & & & & \\
\hline AuE-101 & Ethanol & \multirow{2}{*}{$20-30$ nm } & \multirow{2}{*}{$85 w t \%$} & \multirow{2}{*}{$15 w t \%$} & \\
\hline AuW-101 & Water & & & & \\
\hline
\end{tabular}




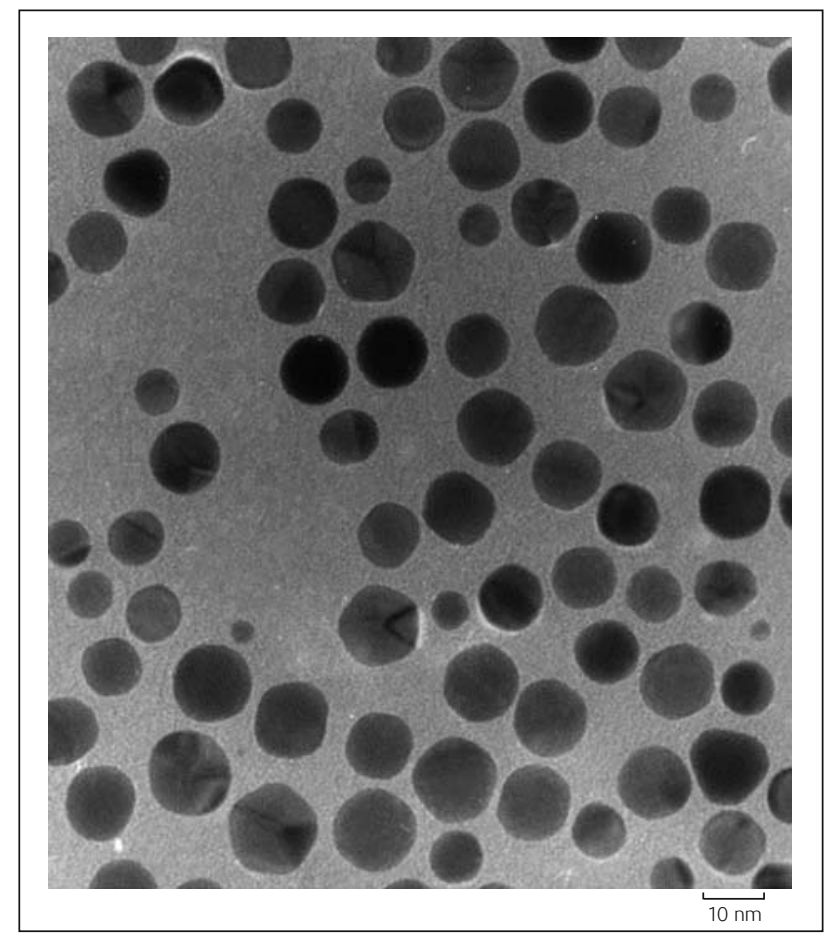

\section{Figure 4}

TEM Image of Gold Nanoparticles

the side chain. Hence, the choice of the side chain allows the polymer to dissolve in various types of solvents. The solvents variation in Table 1 is caused by the polymer that is used. These pastes exhibited neither settling nor coagulation after storage at room temperature for one month.

As can be seen in Table 1, by varying the polymer content in the solid, the gold particle size can be controlled. That is, the average particle size increases when the amount of polymer is reduced. Figure 5 shows the absorption spectrum of the gold nanoparticle paste having particle size of 10 15 $\mathrm{nm}$, as shown in Figure 4. The spectrum is very sharp and the topmost peak was obtained at $525 \mathrm{~nm}$. This implies that the 10 15 nm gold nanoparticle paste shows a distinct red colour.

\subsection{Application as colourants}

i. Gold nanoparticle paint

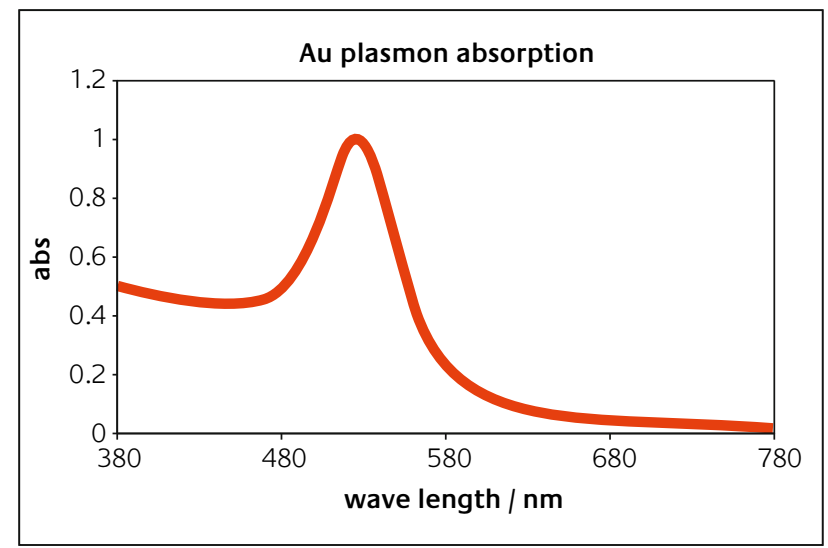

Figure 5

Surface-plasmon Absorption of Gold Nanoparticle paste

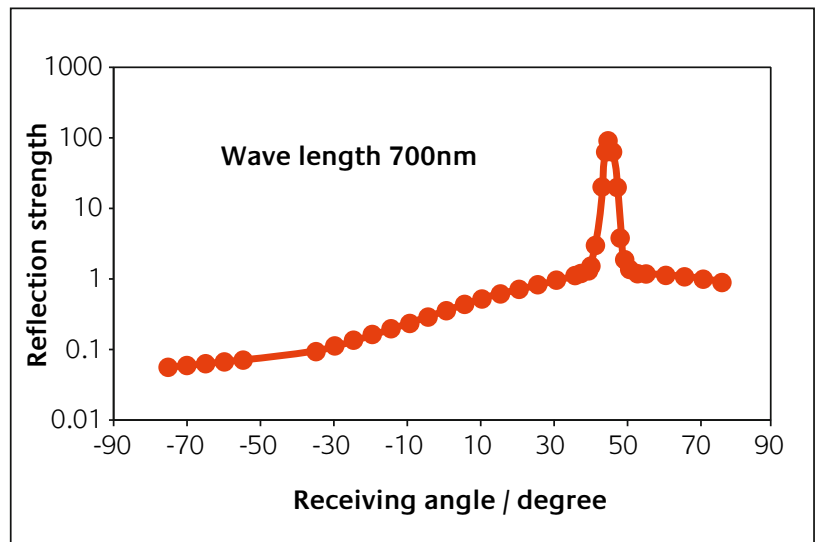

Figure 6

Surface Reflection Strength for a Paint Film Coloured with Gold Nanoparticle

The gold nanoparticle painted panel had an aesthetic appearance, which could not be achieved by ordinary pigments. Moreover, this paint had a higher transparency, as shown in Figure 6. In Figure 6, the angular dependency of the reflected light strength is plotted as a function of the receiving angle. The wave length and the incident angle of light were $700 \mathrm{~nm}$ and -45 degree, respectively. The reflection strength abruptly increased at approximately 45 degree (normal reflection region) and remained less than $1 / 100$ of the peak value at nearly all angles, except at 45 degree (diffusion region). This was due to the particle size that was much smaller than the wave length of light, and the light scattering by gold nanoparticles did not contribute to the reflection. This type of mechanism cannot be observed in ordinary pigmented paint films since the particle size of pigments usually lies between 100 300 nm and light scattering due to pigment particles cannot be ignored. This type of dependency on the angular strength of the reflected light is often referred to as 'flip-flop effect' (7). Furthermore, the colour of this painted panel did not fade for more than 3 years under severe weather conditions in Okinawa.

This paint system could be applied on the automotive model (Figure 7). As can be seen in Figure 7, not only the brightness but also the colour was starkly different in shaded area and highlighted area. We termed this phenomena

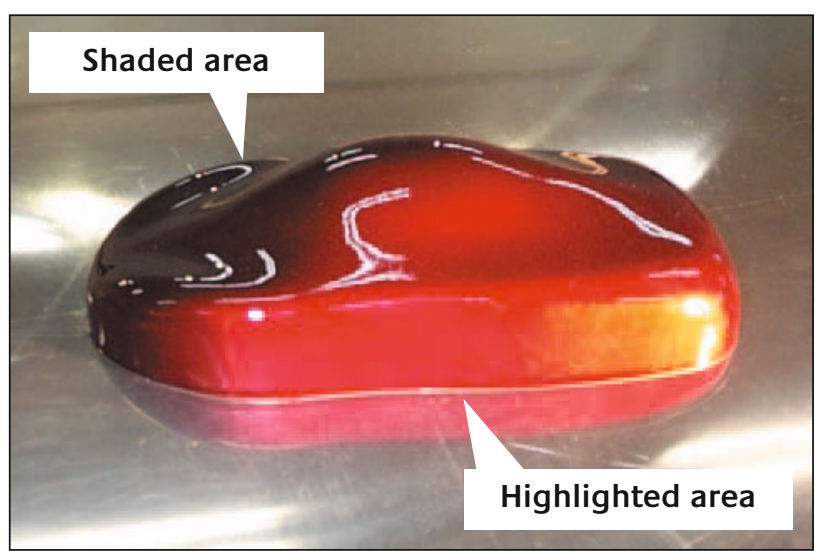

Figure 7

Gold Nanoparticle Paint on Automotive Model 


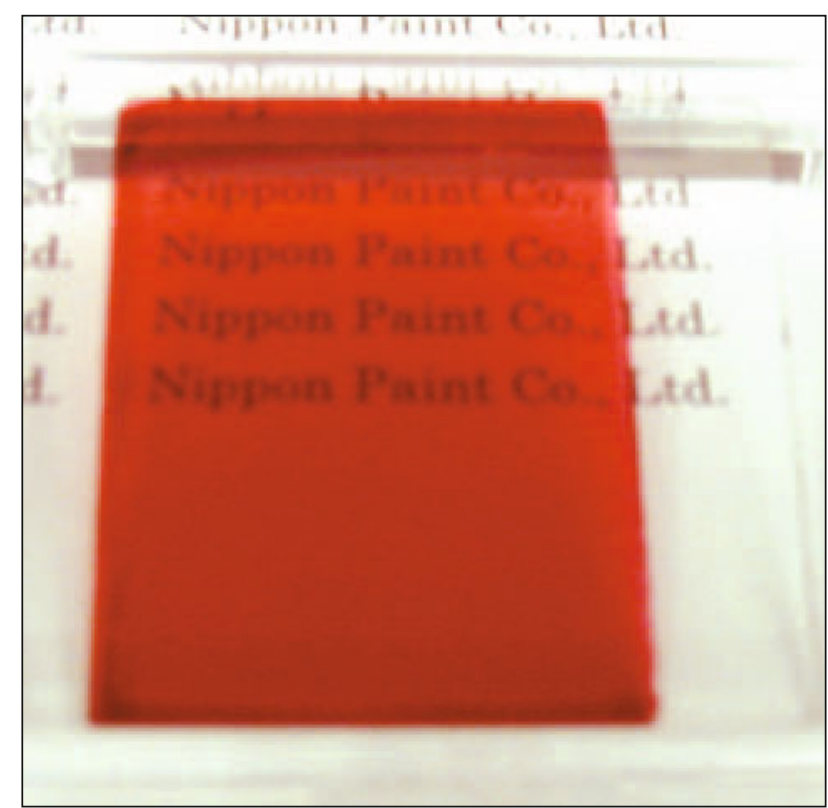

Figure 8

Coloured Glass Film

'colour-flop effect' (8). This effect was due to the surface plasmon absorption. In the highlighted area, we can see the vivid red colour due to the light reflecting from the aluminum flakes. In the shaded area, light did not reflect, hence, the paint appeared to be almost black. Thus, 'colour-flop effect' remarkably enhances the appearance of a rounded shape like a car body.

\section{ii. Coloured glass film}

A coloured glass film that was produced by coating the mixture of gold nanoparticle paste and sol-gel is shown in Figure 8.

Usually sol-gel binder systems exhibit extreme $\mathrm{pH}$ values (9). In these binder systems, it is difficult to maintain the dispersed state of nanosized particles. However, since our gold nanoparticle paste is protected by a special type of protective polymer, it is stable in general sol-gel coating systems without coagulation. This glass film is also stable under severe conditions. This film retained its colour at $280^{\circ} \mathrm{C}$ for $125 \mathrm{~h}$.

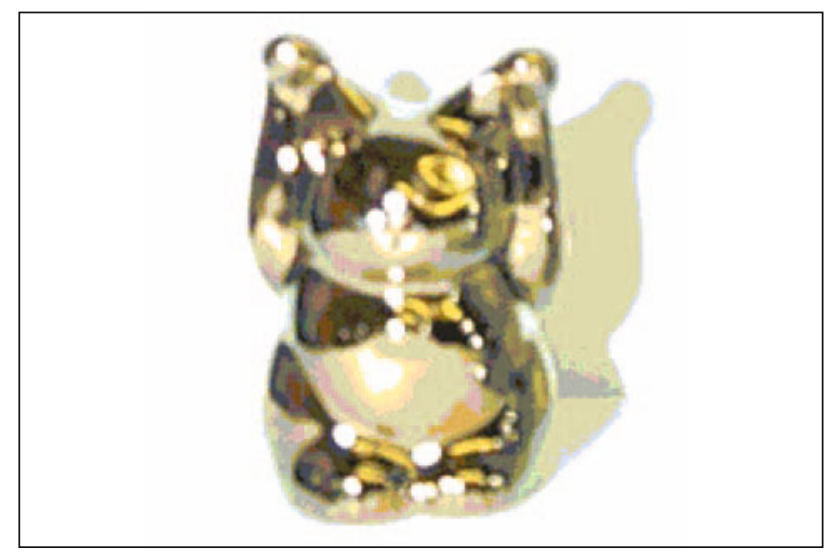

\section{Figure 9}

Thin Gold Film on China Figure

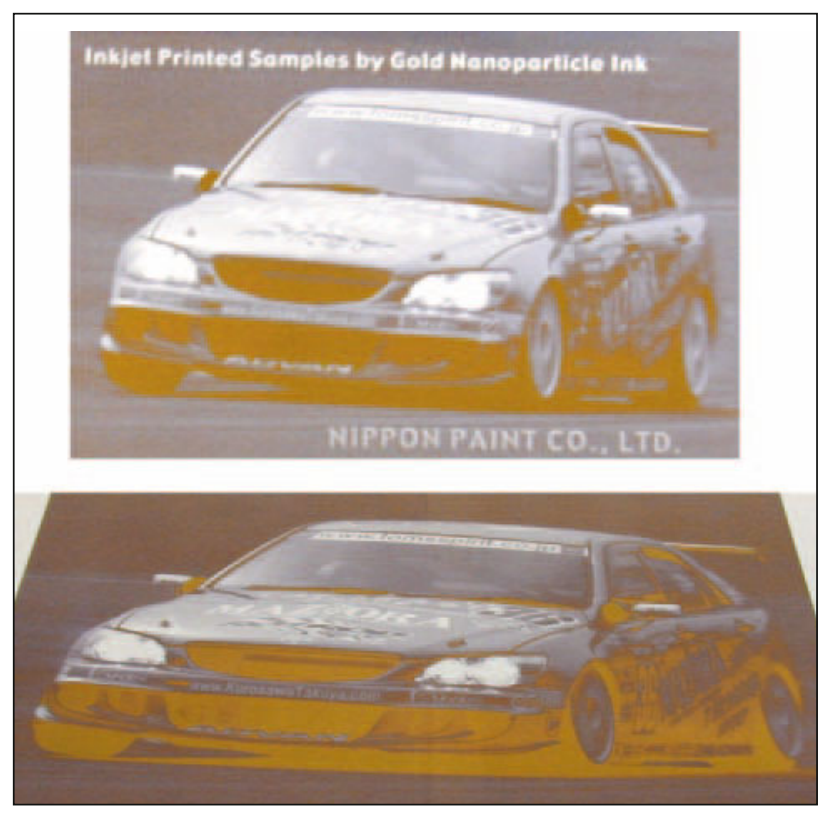

Figure 10

Inkjet Output Pattern using Gold Nanoparticle Ink -Low Dot Density-

\subsection{Application to thin metal film production}

A dried film having a higher gold-content in the solid part of the paste (AuE-101, AuW-101) exhibited a metallic appearance without baking. This could be because the concentration of gold nanoparticles in the film was close to CPVC (10). In the case of the lower gold-content in the solid part of the paste (AuE-001, AuW-001, AuT-001), a gold appearance was observed after baking at $250^{\circ} \mathrm{C}$ (Figure 9). In this case, the protective polymer is partially decomposed and eliminated from the films. In addition to the metallic appearance, these films exhibited electro-conductivity. The elimination of the protective polymer might have caused

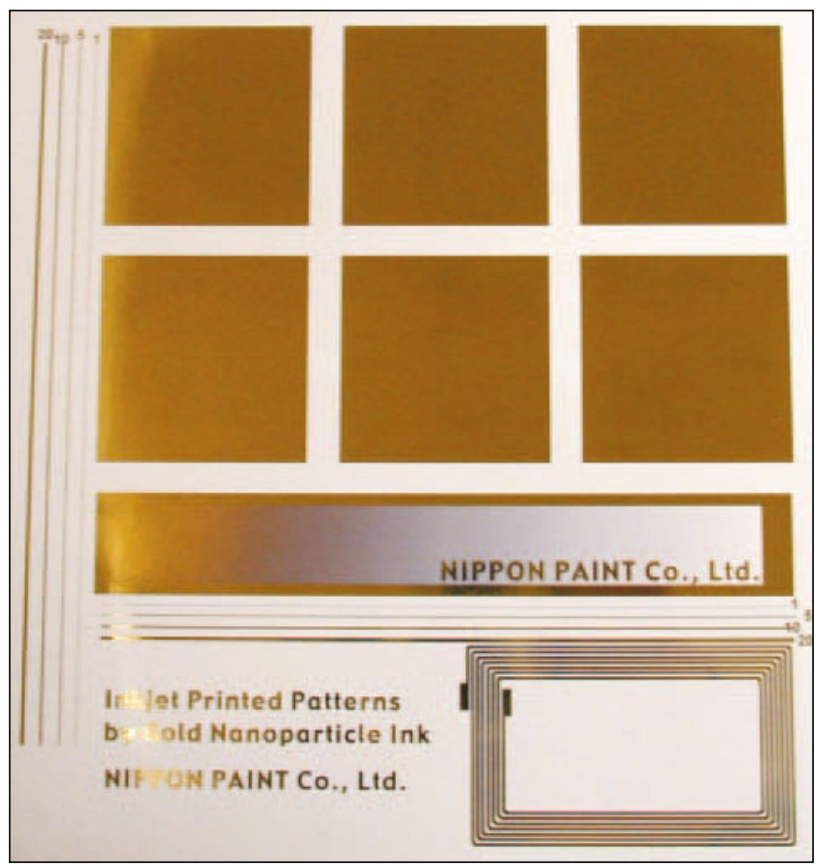

Figure 11

Inkjet Output Pattern using Gold Nanoparticle Ink -High Dot Density- 
coalescence of the gold nanoparticles.

Figure 10 and 11 show the typical output patterns of inkjet printing using the ink containing gold nanoparticle paste. Since the stability of the gold nanoparticle paste is excellent, the ink is applicable to both the thermal-type and piezo-type printers.

When the gold nanoparticle concentration in the ink is low, the output pattern in the case of low dot density mostly exhibits a bluish purple appearance, as shown in Figure 10, while the output pattern in the case of high dot density exhibits a gold appearance for the most part, as shown in Figure 11. When the concentration of gold nanoparticles is high, the output pattern exhibits a gold appearance in all cases of dot density.

\section{Conclusion}

A combination of two key technologies, protection using a comb-shaped block copolymer and reduction by tertiary amine, enabled production of stable and highly concentrated pastes of gold nanoparticles. Thus far, we have succeeded in producing pastes containing up to $85 \mathrm{wt} \%$ gold particles in the solid part. These pastes can be stably diluted or mixed with aqueous and non-aqueous media at arbitrary rates by selecting the polarity of the protective polymer. By using these pastes, we successfully reproduced an aesthetic red colour of stained glass into thin paint films and a glass matrix. The coatings are vivid in colour, have excellent transparency and high durability. We have also successfully produced a very thin and electro-conductive metal film like plating. Although we have described only two applications in this paper, our gold nanoparticle pastes may be applied in various applications in the fields of optics, electronics, catalysis and so on.

\section{$5 \quad$ References}

1 M. Oda, Journal of the Surface Finishing Society of Japan (Hyomen Gijutsu), 1996, 47, 910

2 S. Ogawa, Y. Hayashi, N. Kobayashi, T. Tokizaki, A. Nakamura, Jpn. J. Appl. Phys., 1994, 33, Pt. 2, No. 3A, L331

3 M. Haruta, Catal. Today, 1997, 36, 153 M. Haruta, in GOLD 2003 New Industrial Applications for Gold, 28th September - 1st October 2003, Vancouver A. Cho, Science, 2003, 299, 1684

4 Y. Kurokawa, Y. Hosoya, Surface (Hyomen), 1996, 34, 100 J. Turkevich, G. Garton, P.C. Stevenson, J. Colloid Sci., 1954, 9, 26

5 For example; M.K. Chow, C.F. Zukoski, J. Colloid Interface Sci., 1994, 165, 97 K. Esumi, T. Hosoya, A. Suzuki, K. Torigoe, Langmuir, 2000, 16, 2978 K. Kimura, S. Bandow, Bull. Chem. Soc. Jpn., 1983, 56, 3578 S. Sato, N. Asai, M. Yonese, Colloid Polym. Sci., 1996, 274, 889

6 T. Kobayashi, H. Kamo, Chem. \& Chem. Ind. (Kagaku To Kogyo), 2000,
53, 909

7 S. LeSota, D.J. Engler, J. Landis, R. Johnston-Feller, M.L. McGroarty, J. R. Kiefer, Jr., L. Sander, S. Mark, F.J. Willard, in: PAINT/COATINGS DICTIONARY, Federation of Societies for Coatings Technology, Philadelphia, 1978

F. Sadowski, in ORGANIC COATINGS Science and Technology, Volume 8, G.D. Parfitt, A.V. Patsis (eds.), Marcel Dekker, New York, 1986

8 Z.W. Wicks, Jr., F.N. Jones, S.P. Pappas, in Organic Coatings: Science and Technology, VOLUME I: Film Formation, Components, and Appearance, John Wieley \& Sons, New York, 1992

9 M. Toki, Journal of the Surface Finishing Society of Japan (Hyomen Gijutsu), 1999, 50, 161

J.B. Rosenholm, M. Linden, in HANDBOOK OF Surface and Colloid Chemistry Second Edition, K.S. Birdi (eds.), CRC Press, Boca Raton, 2003

10 CPVC $=$ Critical Pigment Volume Concentration 